\title{
SEQUENCES HAVING AN EFFECTIVE FIXED-POINT PROPERTY $\left({ }^{1}\right)$
}

BY

\author{
T. H. PAYNE
}

\begin{abstract}
Let $\alpha$ be any function whose domain is the set $N$ of all natural numbers. A subset $B$ of $N$ precompletes the sequence $\alpha$ if and only if for every partial recursive function (p.r.f.) $\psi$ there is a recursive function $f$ such that $\alpha f$ extends $\alpha \psi$ and $f[N-$ Dom $\psi] \subset B$. An object $e$ in the range of $\alpha$ completes $\alpha$ if and only if $\alpha^{-1}[\{e\}]$ precompletes $\alpha$. The theory of completed sequences was introduced by A. I. Mal'cev as an abstraction of the theory of standard enumerations. In this paper several results are obtained by refining and extending his methods. It is shown that a sequence is precompleted (by some $B$ ) if and only if it has a certain effective fixed-point property. The completed sequences are characterized, up to a recursive permutation, as the composition $F \varphi$ of an arbitrary function $F$ defined on the p.r.f.'s with a fixed standard enumeration $\varphi$ of the p.r.f.'s. A similar characterization is given for the precompleted sequences. The standard sequences are characterized as the precompleted indexings which satisfy a simple uniformity condition. Several further properties of completed and precompleted sequences are presented, for example, if $B$ precompletes $\alpha$ and $S$ and $T$ are r.e. sets such that $\alpha^{-1}[\alpha[S]] \neq N$ and $\alpha^{-1}[\alpha[T]] \neq N$, then $B-(S \cup T)$ precompletes $\alpha$.
\end{abstract}

1. Preliminaries. Let $\lambda x, y[\langle x, y\rangle]$ be a recursive pairing function which is monotonic in each of its arguments, and let $\rho$ and $\sigma$ denote the corresponding projection functions, $\lambda\langle x, y\rangle[x]$ and $\lambda\langle x, y\rangle[y]$, respectively. For any set $S \subset N$, $\{x \mid\langle i, x\rangle \in S\}$ is called the ith row of $S$ and is denoted by $S_{i}$. The sequence $\lambda i\left[S_{i}\right]$ is called the row sequence of $S$ and is denoted by $S^{*}$. A sequence is said to be recursively enumerable (r.e.) if and only if it is the row sequence of an r.e. set. Every partial function $\psi$ will be identified with its graph $\{\langle x, y\rangle \mid \psi(x)$ is defined and equals $y\}$; hence $\psi^{*}(i)=\psi_{i}=\{\psi(i)\}$ when $i \in \operatorname{Dom} \psi$, and $\psi^{*}(i)=\psi_{i}=\varnothing$ otherwise. (Thus $\psi$ is a p.r.f. if and only if $\psi^{*}$ is an r.e. sequence of sets of cardinality less than two.)

A p.r.f. $\psi$ is called a selector for a set $S \subset N$ if and only if $\psi \subset S$ and Dom $\psi$ $=\left\{i \in N \mid S_{i} \neq \varnothing\right\}$. Note that if $\delta$ is a partial function on $N$ and $\psi$ is a selector for

Received by the editors January 30, 1970 and, in revised form, February 22, 1970.

AMS 1969 subject classifications. Primary 0270; Secondary 0274.

Key words and phrases. Precompleted sequence, completed sequence, effective fixed-point property, recursive isomorphism, recursive reduction, standard sequence, indexing, universal sequence, creative function, universal function.

( ${ }^{1}$ ) This paper is part of the author's doctoral dissertation written during the tenure of a NASA Traineeship at the University of Notre Dame. The author wishes to thank Professor Vladeta Vuckovic for his encouragement and advice.

Copyright (C) 1972, American Mathematical Society 
$\delta^{-1}$ (i.e. $\{\langle y, x\rangle \mid\langle x, y\rangle \in \delta\}$ ), then $\psi$ is $1-1$, Dom $\psi=\operatorname{Rng} \delta$, and $\delta \psi$ is the identity function on Dom $\psi, \operatorname{id}_{\text {Dom } \psi}$. It is well known that every r.e. set $S$ has a selector (e.g. $\lambda x[\sigma f(\mu n[\rho f(n)=x])]$ where $f$ is any recursive function which enumerates $S$ ).

Let $\alpha$ and $\beta$ be sequences or p.r.f.'s. If $f$ is a recursive function (alternately, recursive permutation) such that $\alpha=\beta f$, then we say that $f$ is a reduction (alternately, isomorphism) of $\alpha$ to $\beta$, written $f: \alpha \leqq \beta$ (alternately, $f: \alpha \cong \beta$ ). When such an $f$ exists we say that $\alpha$ is reducible to $\beta$ (alternately, $\alpha$ is isomorphic to $\beta$ ) written $\alpha \leqq \beta$ (alternately, $\alpha \cong \beta$ ).

An r.e. sequence $\alpha$ is universal iff every r.e. sequence whose range is contained in $\operatorname{Rng} \alpha$ is reducible to $\alpha$. We obtain a universal enumeration $W^{*}$ of the r.e. sets by letting $W=\{\langle\langle i, x\rangle, y\rangle \mid\langle x, y\rangle \in \alpha(i)\}$ where $\alpha$ is any r.e. enumeration of the r.e. sets. ( $W^{*}$ is universal since any r.e. sequence must be the row sequence of some $\alpha(i)$, but $\lambda x[\langle i, x\rangle]:(\alpha(i))^{*} \leqq W^{*}$.) We obtain a universal enumeration $\varphi^{*}$ of the p.r.f.'s and a universal enumeration $\Gamma^{*}$ of the sets of cardinality less than two by letting $\Gamma$ be a selector for $\left\{\langle\langle i, x\rangle, y\rangle \mid\langle x, y\rangle \in W_{i}\right\}$ and letting $\varphi=\{\langle i,\langle x, y\rangle\rangle \mid$ $\langle\langle i, x\rangle, y\rangle \in \Gamma\}$. Note that $\varphi_{i}$ is a selector for $W_{i}$ and that $\Gamma=\lambda\langle i, x\rangle\left[\varphi_{i}(x)\right]$. It is easily shown that $\varphi^{*}$ and $\Gamma^{*}$ are universal.

The fact that for every p.r.f. $\psi$ there is a number $n$ such that $\varphi_{n}=\lambda y[\psi\langle n, y\rangle]$ (e.g. $n=\varphi_{m}(m)$ where $\varphi_{m}: \lambda x\left[\lambda y\left[\psi\left\langle\varphi_{x}(x), y\right\rangle\right]\right] \leqq \varphi^{*}$ ) is called the Recursion Theorem.

We let $Q$ denote $N$-Dom $\Gamma, \sim$ denote the equivalence relation induced on $N$ by the sequence $\alpha$, and $\bar{B}$ or $\mathrm{cl}(B)$ denote $\alpha^{-1}[\alpha[B]]$ for any set $B \subset N$. A recursive function $f$ is said to be an $\alpha / B$-extension of a p.r.f. $\psi$ if and only if $\alpha f$ extends $\alpha \psi$ and $f[N-\operatorname{Dom} \psi] \subset B$. B precompletes $\alpha$ if and only if every p.r.f. has an $\alpha / B$-extension. e completes $\alpha$ iff $\alpha^{-1}[\{e\}]$ precompletes $\alpha$. We say that a number $n$ is an $\alpha$-fixed point of a p.r.f. $\psi$ if and only if $n \in \operatorname{Dom} \psi$ and $\psi(n) \sim n$. For any object $e$ and sequence $\beta, E_{\beta}^{e}$ will denote that extension of $\beta \Gamma$ which assigns the value $e$ to every number in $Q$, i.e., $\lambda x[e$ if $x \in Q ; \beta \Gamma(x)$ if $x \notin Q]$.

In [4] Mal'cev showed that every completed sequence $\alpha$ has the effective fixed point property in the sense of Ritter [11], i.e. there exists a recursive function $g$ such that $g(n)$ is an $\alpha$-fixed point of $\varphi_{n}$ whenever $\varphi_{n}$ is total. From the following proposition we see that this property which Mal'cev used as his definition of "precompleted" is equivalent to our present definition of "precompleted."

Proposition 1.1. For any sequence $\alpha$ and set $B \subset N$, the following are equivalent:

(1) For every p.r.f. $\psi$ there is a number $n$ such that $\varphi_{n}$ is an $\alpha / B$-extension of $\lambda x[\psi\langle n, x\rangle]$.

(2) For every p.r.f. $\psi$ there is a $g$ such that $g$ is an $\alpha / B$-extension for $\left.\lambda x\left[\psi_{\langle}^{\prime} g(x), x\right\rangle\right]$.

(3) Every p.r.f. $\psi$ has an $\alpha / B$-extension $g$ (i.e. B precompletes $\alpha$ ).

(4) $\Gamma$ has an $\alpha / B$-extension $g$.

(5) There is a recursive function $g$ such that $g(n)$ is an $\alpha$-fixed point for $\varphi_{n}$ when $\varphi_{n}$ is total, and $g(n) \in B$ when $\varphi_{n}=\varnothing$. 
Proof. (1) implies (2). Substitute $\lambda\langle u, v\rangle\left[\psi\left\langle\varphi_{u}(v), v\right\rangle\right]$ for $\psi$ in (1) and let $g$ be the resulting $\varphi_{n}$.

(2) implies (3). Let $\psi$ be any p.r.f. Substitute $\lambda\langle u, v\rangle[\psi(v)]$ for $\psi$ in (2). Then the resulting $g$ is an $\alpha / B$-extension of $\psi$.

(3) implies (4). Obvious.

(2) implies (5). Substitute $\lambda\langle u, v\rangle\left[\varphi_{u}(v)\right]$ for $\psi$ in (2).

(5) implies (4). Suppose (5) holds. Let $h: \lambda x[\lambda y[\Gamma(x)]] \leqq \varphi^{*}$. If $\Gamma(n)$ is defined, $\varphi_{h(n)}$ is the constant function whose range is $\{\Gamma(n)\}$ and hence $\varphi_{h(n)}$ is total and

$$
g h(n) \sim \varphi_{h(n)}(g h(n))=\Gamma(n)
$$

(since $g h(n)$ is an $\alpha$-fixed point of $\left.\varphi_{h(n)}\right)$. If, however, $\Gamma(n)$ is not defined, then $\varphi_{h(n)}=\varnothing$, so $g h(n) \in B$. Thus $g h$ is an $\alpha / B$-extension of $\Gamma$.

(4) implies (3). Suppose that $f$ is an $\alpha / B$-extension of $\Gamma$ and that $\psi$ is any p.r.f. By the universality of $\Gamma^{*}$ there is a recursive function $g$ such that $\psi=\Gamma g$. Thus, $f g$ is an $\alpha / B$-extension of $\psi$.

(3) implies (1). Let $\psi$ be any p.r.f., and let $f$ be an $\alpha / B$-extension of $\psi$. By the Recursion Theorem we may choose $n$ so that $\varphi_{n}$ equals $\lambda x[f\langle n, x\rangle]$ which is clearly an $\alpha / B$-extension of $\lambda x[\psi\langle n, x\rangle]$.

LEMmA 1.2. Suppose that $\beta$ extends $\alpha \Gamma$. Then any selector $f$ for $\Gamma^{-1}$ is $1-1$ and reduces $\alpha$ to $\beta$ (for $\alpha=\alpha \Gamma f=\beta f$, since $\left.\Gamma f=\mathrm{id}_{\mathrm{Rng} \Gamma}=\mathrm{id}_{N}\right)$. Furthermore, if $g: \beta \leqq \alpha$, then $g$ is an $\alpha / g[Q]$-extension of $\Gamma$ and hence $g[Q]$ precompletes $\alpha$.

2. Other sets which precomplete $\alpha$. Suppose that $B$ precompletes $\alpha$. Clearly every superset of $B$ precompletes $\alpha$. The following results show us some of the subsets of $B$ which precomplete $\alpha$, as well as showing that certain kinds of r.e. sequences cannot cover $B$.

LEMMA 2.1. If B precompletes $\alpha$ and $\psi$ is a p.r.f. having no $\alpha$-fixed points, then $B$-Dom $\psi$ precompletes $\alpha$.

Proof. Let $\delta$ be a p.r.f. such that $\delta\langle x, y\rangle$ is either $\Gamma(x)$ or $\psi(y)$ when at least one of these is defined, and otherwise $\delta\langle x, y\rangle$ is undefined. That is, let $\delta$ be a selector for $\{\langle\langle x, y\rangle, z\rangle \mid z=\Gamma(x)$ or $z=\psi(y)\}$. With the aid of 1.1 part (2) choose $g$ so that $g$ is an $\alpha / B$-extension of $\lambda x[\delta\langle x, g(x)\rangle]$. Notice that if $x \in \operatorname{Dom} \Gamma$, then $\langle x, g(x)\rangle$ $\in$ Dom $\delta$. However, if $\langle x, g(x)\rangle \in \operatorname{Dom} \delta$, then $\delta\langle x, g(x)\rangle \neq \psi(g(x))$ (hence $x \in$ Dom $\Gamma$ and $\delta\langle x, g(x)\rangle=\Gamma(x))$ since $\delta\langle x, g(x)\rangle \sim g(x)$ by our choice of $g$, while $g(x) \nsim \psi(g(x))$ since $\psi$ has no $\alpha$-fixed points. Thus, if $x \in \operatorname{Dom} \Gamma$ then $g(x)$ $\sim \delta\langle x, g(x)\rangle=\Gamma(x)$. Conversely, if $x \notin \operatorname{Dom} \Gamma$, then $\langle x, g(x)\rangle \notin$ Dom $\delta$, and hence $g(x) \in B$ and $g(x) \notin$ Dom $\psi$ since $N \times$ Dom $\psi \subset$ Dom $\delta$. Thus $g$ is an $\alpha /(B-\operatorname{Dom} \psi)$ extension of $\Gamma$, so by 1.1 part (4), $B$-Dom $\psi$ precompletes $\alpha$.

Corollary 2.2. Suppose that $B$ precompletes $\alpha$ and $e \in \operatorname{Rng} \alpha$.

(a) Let $S^{*}$ be an r.e. sequence and $\delta$ be a p.r.f. such that $i \in \operatorname{Dom} \delta$ and $\delta(i) \notin \operatorname{cl}\left(S_{i}\right)$ whenever $S_{i} \neq \varnothing$. Then $B-\bigcup\left\{S_{i} \mid i \in N\right\}$ precompletes $\alpha$. (Note that in such a case $B \nsubseteq \bigcup\left\{S_{i} \mid i \in N\right\}$.) 
(b) Let $S_{0}$ and $S_{1}$ be r.e. If $\mathrm{cl}\left(S_{0}\right) \neq N$ and $\mathrm{cl}\left(S_{1}\right) \neq N$, then $B-\left(S_{0} \cup S_{1}\right)$ precompletes $\alpha$. (Thus, if $B \subset S_{0} \cup S_{1}$, then $\mathrm{cl}\left(S_{0}\right)=N$ or $\mathrm{cl}\left(S_{1}\right)=N$. In particular, if $B \subset S_{1}$, then $\operatorname{cl}\left(S_{1}\right)=N$.)

(c) Let $N-\alpha^{-1}[\{e\}]$ be r.e. Then e completes $\alpha$ and only e completes $\alpha$.

Proof. (a) Let $\psi$ be a selector for $\left\{\langle x, y\rangle \mid x \in S_{i}\right.$ and $y=\delta(i)$ for some $i \in$ Dom $\left.\delta\right\}$. Then $\psi$ has no $\alpha$-fixed points and Dom $\psi=\bigcup\left\{S_{i} \mid i \in N\right\}$. Thus, by Lemma 2.1, $B-\bigcup\left\{S_{i} \mid i \in N\right\}$ precompletes $\alpha$.

(b) Choose $\delta$ so that $\delta(0) \notin \mathrm{cl}\left(S_{0}\right)$ and $\delta(1) \notin \mathrm{cl}\left(S_{1}\right)$, and apply (a).

(c) $\operatorname{cl}\left(N-\alpha^{-1}[\{e\}]\right) \neq N$ since $e \in \operatorname{Rng} \alpha$. Thus, by (b), $B \cap \alpha^{-1}[\{e\}]$ precompletes $\alpha$, hence $\alpha^{-1}[\{e\}]$ precompletes $\alpha$, hence $e$ completes $\alpha$. Now if $e^{\prime}$ completes $\alpha$ and $e^{\prime} \neq e$, then $\alpha^{-1}\left[\left\{e^{\prime}\right\}\right] \subset N-\alpha^{-1}[\{e\}]$, hence $\operatorname{cl}\left(N-\alpha^{-1}[\{e\}]\right)=N$ (a contradiction).

3. Isomorphisms. By extending the methods of Myhill [6] and Rogers [8], Mal'cev proved the following lemma which has the isomorphism theorems of [1], [2], [6], [8], [9] and [11] as corollaries. This result also follows from the main theorem of Ritter [7].

LEMMA 3.1. If $\alpha$ is precompleted, then $\beta \cong \alpha$ whenever $\beta \leqq \alpha$ and $\alpha \leqq \beta$.

In this section we refine these methods somewhat in order to obtain the following lemma which is needed for our characterization theorems.

LEMMA 3.2. B precompletes $\alpha$ iff $\Gamma$ has an $\alpha / B$-extension which is a permutation on $N$. (In particular, e completes $\alpha$ iff $\alpha$ is isomorphic to $E_{\alpha}^{e}$.)

The proofs of 3.1 and 3.2 require the following preliminary lemmas.

Lemma 3.3. (Simultaneous Definitions.) Let $O_{1}, \ldots, O_{p}$ be effective $p$-ary operations on the class of partial functions. Suppose that $n \leqq p$ and for all $i \in\{1, \ldots, n\}, B_{i}$ precompletes $\alpha_{i}$. Then there exist p.r.f.'s $\delta_{1}, \ldots, \delta_{p}$ such that

$$
\begin{aligned}
& \delta_{1} \text { is an } \alpha_{1} / B_{1} \text {-extension of } O_{1}\left(\delta_{1}, \ldots, \delta_{p}\right) \\
& \vdots \\
& \delta_{n} \text { is an } \alpha_{n} / B_{n} \text {-extension of } O_{n}\left(\delta_{1}, \ldots, \delta_{p}\right) \\
& \delta_{n+1}=O_{n+1}\left(\delta_{1}, \ldots, \delta_{p}\right) \\
& \vdots \\
& \delta_{p}=O_{p}\left(\delta_{1}, \ldots, \delta_{p}\right) .
\end{aligned}
$$

Proof. Let $\psi_{i}$ be an $\alpha_{i} / B_{i}$-extension of (alternately, be equal to)

$$
\lambda\langle x, y\rangle\left[O_{i}\left(\varphi_{\varphi_{x}(1)}, \ldots, \varphi_{\varphi_{x}(p)}\right)(y)\right]
$$

for $i=1, \ldots, n$ (alternately, for $i=n+1, \ldots, p)$. Let $f: \lambda\langle x, i\rangle\left[\lambda y\left[\psi_{i}\langle x, y\rangle\right]\right] \leqq \varphi^{*}$. Using the Recursion Theorem, choose $m$ so that $\varphi_{m}=\lambda i[f\langle m, i\rangle]$. Let $\delta_{i}=\varphi_{\varphi_{m}(i)}$ for $i=1, \ldots, p$. Then $\delta_{i}=\lambda y\left[\psi_{i}\langle m, y\rangle\right]$ which is an $\alpha_{i} / B_{i}$-extension of (alternately, equal to) $O_{i}\left(\varphi_{\varphi_{m}(1)}, \ldots, \varphi_{\varphi_{m}(p)}\right)$, i.e. $O_{i}\left(\delta_{1}, \ldots, \delta_{n}\right)$, when $1 \leqq i \leqq n$ (alternately, when $n+1 \leqq i \leqq p)$. 
LEMMA 3.4. Suppose that $f$ is a recursive function, that $\alpha(x)=\alpha(y)$ whenever $f(x)=f(y)$, and that $f$ is 1-1 if $\alpha$ is constant. Then the five parts of Proposition 1.1 are equivalent to the stronger conditions obtained when the requirement that $f g$ be strictly monotonic is added to parts (2)-(5) and the requirement that $f \varphi_{n}$ be strictly monotonic is added to part (1). (In particular, we may require that $g$ and $\varphi_{n}$ be monotonic by letting $f$ be the identity function on $N$.)

Proof. The case where $\alpha$ is constant is trivial. Suppose that $\alpha$ is nonconstant, that $B$ precompletes $\alpha$, and that $f$ is a recursive function such that $\alpha(x)=\alpha(y)$ whenever $f(x)=f(y)$. Let $\psi$ be any p.r.f. We begin by showing that $\psi$ has an $\alpha / B$-extension $h$ such that $f h$ is strictly monotonic. Let $m_{1}$ and $m_{2}$ be numbers such that $\alpha\left(m_{1}\right)$ $\neq \alpha\left(m_{2}\right)$. Let $D$ be the canonical enumeration of the finite sets (cf., Rogers [10, p. 70]). By simultaneous definition there exist a recursive function $k$ and a p.r.f. $h$ such that $k$ is an $\alpha / B$-extension of

$$
\begin{aligned}
\lambda\langle n, x\rangle\left[m_{2}\right. & \text { if } f k\langle n, x\rangle \\
m_{1} \text { if } f k\langle n, x\rangle & \left.\in\{f h(0), \ldots, f h(n-1), \ldots, f h(n-1)\}-D_{x} ; \psi \rho(n) \text { otherwise }\right]
\end{aligned}
$$

and $h=\lambda n[k\langle n, \mu x[f k\langle n, x\rangle \notin\{f h(0), \ldots, f h(n-1)\}]\rangle]$.

Assume that $h(0), \ldots, h(n-1)$ are defined and $f h(0), \ldots, f h(n-1)$ are distinct. Let $D_{x}=\left\{f h(i) \mid \alpha h(i)=\alpha\left(m_{1}\right)\right.$ and $\left.i<n\right\}$.

Suppose that $f k\langle n, x\rangle=f h(j)$ for some $j<n$. Then, by our assumption about $f$, $\alpha h(j)=\alpha k\langle n, x\rangle$. Suppose that $f h(j) \in D_{x}$. Then $\alpha h(j)=\alpha\left(m_{1}\right)$ by the definition of $D_{x}$, while $\alpha k\langle n, x\rangle=\alpha\left(m_{2}\right)$ by the definition of $k$. Hence $\alpha\left(m_{1}\right)=\alpha\left(m_{2}\right)$ which contradicts our assumption that $\alpha\left(m_{1}\right) \neq \alpha\left(m_{2}\right)$. Thus, $f h(j) \notin D_{x}$. Hence $\alpha h(j) \neq \alpha\left(m_{1}\right)$ by the definition of $D_{x}$, while $\alpha k\langle n, x\rangle=\alpha\left(m_{1}\right)$ by the definition of $k$. This is a contradiction; hence no such $j$ can exist.

Thus $f k\langle n, x\rangle \notin\{f h(0), \ldots, f h(n-1)\}$. Let $u$ denote

$$
\mu x[f k\langle n, x\rangle \notin\{f h(0), \ldots, f h(n-1)\}] .
$$

Then, $h(n)=k\langle n, u\rangle \sim \psi \rho(n)$ when $\psi \rho(n)$ is defined, and $h(n)=k\langle n, u\rangle \in B$ otherwise.

By induction we see that $h$ is an $\alpha / B$-extension of $\psi \rho$ and $f h$ is $1-1$. But $\rho$ $=\lambda\langle n, x\rangle[n]$, hence $\rho$ assumes each value infinitely often. Thus one can easily find an $\alpha / B$-extension $h^{\prime}$ of $\psi$ such that $f h^{\prime}$ is strictly monotonic, e.g.,

$$
\lambda n\left[h\left\langle n, \mu x\left[f h\langle n, x\rangle>f h^{\prime}(0) \& \cdots \& f h\langle n, x\rangle>f h^{\prime}(n-1)\right]\right\rangle\right] .
$$

An inspection of the proof of Proposition 1.1 shows that the stronger forms of (1), (2), (4), and (5) follow from the stronger form of (3) which we have just proved.

LEMMA 3.5. Suppose that $f$ is a 1-1 recursive function and that $g$ is a strictly monotonic recursive function. Then there is a recursive permutation $p$ such that $p(x)=g(x)$ whenever $x \notin \operatorname{Rng} f$ and such that $p: \alpha \cong \beta$ whenever $g: \alpha \leqq \beta$ and $f: \beta \leqq \alpha$. (More generally, we can allow $g$ to be any 1-1 recursive function having $a$ recursive range.) 
This lemma is proved in the same way as Myhill's theorem [10, p. 85].

Proof of 3.1. Suppose that $\alpha$ is precompleted. Let $g: \alpha \leqq \beta$ and $f: \beta \leqq \alpha$. By Lemma 3.4, $f$ has a $1-1 \alpha / N$-extension $f^{\prime}$; obviously $f^{\prime}: \alpha \leqq \beta$. It is evident that $\alpha(x)=\alpha(y)$ whenever $g(x)=g(y)$, since $g: \alpha \leqq \beta$. Thus by Lemma 3.4, $\mathrm{id}_{N}$ has an $\alpha / B$-extension $h$ such that $g h$ is strictly monotonic; clearly $g h: \alpha \leqq \beta$. Thus by applying Lemma 3.5 to $g h$ and $f^{\prime}$ we see that there is a $p$ such that $p: \alpha \cong \beta$.

Proof of Lemma 3.2. If $\Gamma$ has an $\alpha / B$-extension which is a recursive permutation, then $B$ precompletes $\alpha$ by Proposition 1.1 part (4).

Suppose that $B$ precompletes $\alpha$. Let $g$ be a monotonic $\alpha / B$-extension of $\Gamma$, and let, "be a selector for $\Gamma^{-1}$. Then, by Lemma $1.2, f$ is $1-1$ and $f: \alpha \leqq \alpha g$. But clearly $g: \alpha g \leqq \alpha$. Thus, by Lemma 3.5, there exists $p$ such that $p: \alpha g \cong \alpha$ and such that $p(x)$ $=g(x)$ whenever $x \in N-\operatorname{Rng} f$. But $Q=N-\operatorname{Dom} \Gamma \subset N-\operatorname{Rng} f$; so $p[Q]=g[Q]$ $\subset B$ since $g$ is an $\alpha / B$-extension of $\Gamma$.

4. Related sequences. A sequence $\beta$ reducible to $\alpha$ is said to be $\alpha$-universal iff $\gamma \leqq \beta$ whenever $\gamma \leqq \alpha$ and $\operatorname{Rng} \gamma \subset \operatorname{Rng} \beta$. Clearly a sequence is $W^{*}$-universal iff it is universal.

A sequence $\beta$ reducible to $\alpha$ is said to be a (sub)retract of $\alpha$ iff there exists a (partial) recursive function $\psi$, called a (sub)retraction of $\alpha$ to $\beta$, such that $\psi(x)$ is defined and $\beta \psi(x)=\alpha(x)$ whenever $\alpha(x) \in \operatorname{Rng} \beta$; in such a case $\beta$ is $\alpha$-universal, for if $f: \gamma \leqq \alpha$ and $\operatorname{Rng} \gamma \subset \operatorname{Rng} \beta$ then $\psi f: \gamma \leqq \beta$. Notice that any isomorphism from $\alpha$ to $\beta$ is a retraction of $\alpha$ to $\beta$.

A recursive permutation $p$ for which there is a function $F$ from $\operatorname{Rng} \alpha$ to $\operatorname{Rng} \beta$ such that $p: F \alpha \cong \beta$ is said to be a homomorphism from $\alpha$ to $\beta$, written $p: \alpha \rightarrow \beta$; in such a case $\beta$ is said to be a homomorphic image of $\alpha$ and $F$ is called the induced function. Notice that there can be at most one such $F$ and that $\operatorname{id}_{N}: \alpha \rightarrow \beta$ iff $\beta(x)$ $=\beta(y)$ whenever $\alpha(x)=\alpha(y)$. Clearly any sequence isomorphic to a homomorphic image of $\alpha$ is also a homomorphic image of $\alpha$.

LEMMA 4.1 (MAL'CEV). If a class $\mathscr{S}$ contained in $\mathrm{Rng} \alpha$ has a precompleted $\alpha$-universal enumeration, then the $\alpha$-universal enumerations of $\mathscr{S}$ constitute an isomorphism class.

Proof. Suppose $\beta$ is a precompleted $\alpha$-universal enumeration of $\mathscr{S}$. Clearly, if $\gamma \cong \beta$, then $\gamma$ is an $\alpha$-universal enumeration of $\mathscr{S}$. Conversely, if $\gamma$ is an $\alpha$-universal enumeration of $\mathscr{S}$, then $\gamma \leqq \beta$ and $\beta \leqq \gamma$, and hence $\gamma \cong \beta$ by Lemma 3.1.

LEMMA 4.2 (MAL'CEv). $\beta$ is a subretract of $\alpha$ iff $\beta$ is uniformly $\alpha$-universal in the sense that there exists a recursive function $g$ such that if $\operatorname{Rng} \gamma \subset \operatorname{Rng} \beta$ and $\varphi_{n}: \gamma \leqq \alpha$, then $\varphi_{g(n)}: \gamma \leqq \beta$.

Proof. Suppose $\psi$ is a subretraction of $\alpha$ to $\beta$. Let $g: \lambda n\left[\lambda x\left[\psi \varphi_{n}(x)\right]\right] \leqq \varphi^{*}$. If Rng $\gamma \subset \operatorname{Rng} \beta$ and $\varphi_{n}: \gamma \leqq \alpha$, then $\beta \varphi_{g(n)}=\beta \psi \varphi_{n}=\alpha \varphi_{n}=\gamma$ and hence $\varphi_{g(n)}: \gamma \leqq \beta$. Thus $\beta$ is uniformly $\alpha$-universal. 
Conversely, suppose that $\beta$ is uniformly $\alpha$-universal via $g$. Let $\psi=\lambda n\left[\varphi_{g h(n)}(1)\right]$ where $h: \lambda n[\lambda x[n]] \leqq \varphi^{*}$. Suppose that $\alpha(n) \in \operatorname{Rng} \beta$. Then $\operatorname{Rng} \alpha \varphi_{h(n)}=\{\alpha(n)\}$ $\subset \operatorname{Rng} \beta$ and $\varphi_{h(n)}: \alpha \varphi_{h(n)} \leqq \alpha$; thus $\varphi_{g h(n)}: \alpha \varphi_{h(n)} \leqq \beta$; hence $\psi(n)$ is defined and $\alpha(n)$ $=\alpha \varphi_{h(n)}(1)=\beta \varphi_{g h(n)}(1)=\beta \psi(n)$. Thus $\psi$ is a subretraction of $\alpha$ to $\beta$.

LEMма 4.3. Every precompleted subretract of $\alpha$ is a retract of $\alpha$. Furthermore, when $\alpha$ is precompleted, so is every retract of $\alpha$.

Proof. Let $\beta$ be precompleted and $\psi$ be a subretraction of $\alpha$ to $\beta$. Let $f$ be a $\beta / N$-extension of $\psi$. If $\alpha(x) \in \operatorname{Rng} \beta$, then $\psi(x)$ is defined and $\beta f(x)=\beta \psi(x)=\alpha(x)$. Thus $f$ is a retraction of $\alpha$ to $\beta$.

The second part of this lemma is a corollary to Lemma 4.4 part (c) (below).

Lemma 4.4. Let B precomplete $\alpha$ (respectively, let e complete $\alpha$ ):

(a) If $\beta \leqq \alpha$, then there is a recursive function $h$ such that $\alpha$ extends $\beta \Gamma$ and $h[Q] \subset B$ (respectively, $\left.h: E_{\beta}^{e} \leqq \alpha\right)$.

(b) If $\beta$ is $\alpha$-universal and $\alpha[B] \subset \operatorname{Rng} \beta$ (respectively, $e \in \operatorname{Rng} \beta$ ), then $\beta^{-1}[\alpha[B]]$ precompletes $\beta$ (respectively, e completes $\beta$ ).

(c) If $\psi$ is a subretraction of $\alpha$ to $\beta$ whose domain includes $B$, then $\psi[B]$ precompletes $\beta$.

(d) If $f: \alpha \rightarrow \beta$, then $f[B]$ precompletes $\beta$ (respectively, the induced image of $e$ completes $\beta$ ).

Proof. (a) Suppose that $g: \beta \leqq \alpha$. Let $h$ be an $\alpha / B$-extension of $g \Gamma$. If $\Gamma(x)$ is defined, then $\beta \Gamma(x)=\alpha g \Gamma(x)=\alpha h(x)$. Thus $\alpha h$ extends $\beta \Gamma$. If $\Gamma(x)$ is not defined (i.e. if $x \in Q)$, then $h(x) \in B$. Thus $h[Q] \subset B$.

(b) Suppose that $\beta$ is $\alpha$-universal and $\alpha[B] \subset \operatorname{Rng} \beta$. Let $h$ be as in (a). Then $\mathrm{Rng}$ $\alpha h \subset \operatorname{Rng} \beta$. Thus $\alpha h \leqq \beta$, say $g: \alpha h \leqq \beta$. Then $g[Q]$ precompletes $\beta$, by Lemma 1.2. But $g[Q] \subset \beta^{-1}[\alpha h[Q]] \subset \beta^{-1}[\alpha[B]]$. So $\beta^{-1}[\alpha[B]]$ precompletes $\beta$.

(c) Suppose that $\psi$ is a subretraction of $\alpha$ to $\beta$ and that $B \subset$ Dom $\psi$. Let $h$ be as in (a). Then $\psi h: \alpha h \leqq \beta$. Thus, by Lemma $1.2, \psi h[Q]$ precompletes $\beta$. But $h[Q] \subset B$; so $\psi[B]$ precompletes $\beta$.

(d) Let $f: F \alpha \cong \beta$. Clearly $B$ precompletes $F \alpha$ since any $\alpha / B$-extension of $\Gamma$ is an $F \alpha / B$-extension of $\Gamma$. Thus $f[B]$ precompletes $\beta$, by (c), since $f$ is an isomorphism and, hence, a retraction from $F \alpha$ to $\beta$.

COROLlary 4.5. $\varnothing$ completes $W^{*}, \varphi^{*}$, and $\Gamma^{*}$.

Proof. $\lambda i\left[W_{\Gamma(i)}\right.$ if $i \in \operatorname{Dom} \Gamma$; $\varnothing$ otherwise $]$ is r.e. since it is the row sequence of $\{\langle i, x\rangle \mid$ for some $y,\langle i, y\rangle \in \Gamma$ and $\langle y, x\rangle \in W\}$. Let $f$ reduce it to $W^{*}$. Then $f$ is a $W^{*} /\left(W^{*}\right)^{-1}[\{\varnothing\}]$-extension of $\Gamma$. Hence $\varnothing$ completes $W^{*}$. Thus, by $4.4(\mathrm{~b}), \varnothing$ also completes $\varphi^{*}$ and $\Gamma^{*}$ since they are $W^{*}$-universal and $\varnothing$ is a member of their ranges.

COROLlaRY 4.6. For any sequence $\alpha, \mathrm{id}_{N}: \Gamma^{*} \rightarrow E_{\alpha}^{e}$ with $e$ as the induced image of $\varnothing$; hence e completes $E_{\alpha}^{e}$. 
Proof. Suppose $\Gamma^{*}(x)=\Gamma^{*}(y)$. If $x \in \operatorname{Dom} \Gamma$, then $\{\Gamma(x)\}=\Gamma^{*}(x)=\Gamma^{*}(y)$ $=\{\Gamma(y)\}$, hence $E_{\alpha}^{e}(x)=\alpha \Gamma(x)=\alpha \Gamma(y)=E_{\alpha}^{e}(y)$. If $x \notin$ Dom $\Gamma$, then $\Gamma^{*}(x)=\varnothing$ $=\Gamma^{*}(y)$, hence $y \notin \operatorname{Dom} \Gamma$, so $E_{\alpha}^{e}(x)=e=E_{\alpha}^{e}(y)$. Thus $\mathrm{id}_{N}: \Gamma^{*} \rightarrow E_{\alpha}^{e}$, and $E_{\alpha}^{e}(x)=e$ whenever $\Gamma^{*}(x)=\varnothing$.

\section{Creative functions.}

Lemma 5.1. Let $B$ precomplete $\alpha$ and let $F$ be a function such that $F \alpha$ is a p.r.f. Then either $\varnothing$ completes $(F \alpha)^{*}$ (in which case $(F \alpha)^{*}$ is universal) or $F \alpha$ is total and constant.

Proof. Clearly id ${ }_{N}: \alpha \rightarrow(F \alpha)^{*}$. So, by Lemma 4.4 part (d), $B$ precompletes $(F \alpha)^{*}$. Suppose $F \alpha$ is not constant or not total. In the case where $F \alpha$ is not constant let $\delta$ be a selector for the set whose row sequence is $\lambda i[\{x \mid F \alpha(x) \neq i\}]$, and in the case where $F \alpha$ is not total let $\delta=\lambda x[n]$ where $n \notin$ Dom $F \alpha$. By applying Lemma 2.2 to $\delta$ and the sequence $S^{*}=\lambda i[\{x \mid F \alpha(x)=i\}]$ we see that $B-\bigcup\left\{S_{i} \mid i \in N\right\}$ precompletes $\alpha$. But $B-\bigcup\left\{S_{i} \mid i \in N\right\}=B-\operatorname{Dom} F \alpha \subset\left((F \alpha)^{*}\right)^{-1}[\{\varnothing\}]$; hence $\varnothing$ completes $\alpha$.

Now let $\eta$ be any p.r.f. whose range is contained in Rng $F \alpha$ (i.e. $\eta^{*}$ be any r.e. sequence whose range is contained in $\left.\operatorname{Rng}(F \alpha)^{*}\right)$. Let $g$ be an $(F \alpha)^{*} /(B-\operatorname{Dom} F \alpha)$ extension of $\psi \eta$ where $\psi$ is any selector for $(F \alpha)^{-1}$. Now if $\eta(x)$ is defined, then $F \alpha g(x)=F \alpha \psi \eta(x)=\eta(x)$ since $F \alpha \psi$ is the identity function on the range of $F \alpha$ which contains the range of $\eta$. On the other hand, if $\eta(x)$ is not defined, then $F \alpha g(x)$ is not defined since $g(x) \in B-\operatorname{Dom} F \alpha$. Hence $g: \eta \leqq F \alpha$; so $g: \eta^{*} \leqq(F \alpha)^{*}$. Thus $(F \alpha)^{*}$ is universal.

Proposition 5.2. Let $R$ be an r.e. set. For any p.r.f. $\psi$ the following are equivalent:

(1) $\psi$ is isomorphic to the restriction, $\left.\Gamma\right|_{\Gamma^{-1}[R]}$, of $\Gamma$ to $\Gamma^{-1}[R]$.

(2) $\psi^{*}$ is a universal enumeration of the class of all subsets of $R$ having cardinality less than two.

(3) $\psi$ is maximal with respect to $\leqq$ in the class of all p.r.f.'s whose ranges are contained in $R$.

(4) $\psi$ is creative and $\operatorname{Rng} \psi=R$ (cf. Cleave [1] and Lachlan [2]).

(5) $\left.\Gamma\right|_{\Gamma^{-1}[R]} \leqq \psi$ and $\operatorname{Rng} \psi=R$.

(6) $\varnothing$ completes $\psi^{*}$ and $\mathrm{Rng} \psi=R$ (cf. Mal'cev [4]).

(7) $\psi^{*}$ is precompleted, $\operatorname{Rng} \psi=R$, and $\psi$ is not constant and total.

Furthermore, when $R=N$ we may add:

(8) $\psi$ is universal in the sense of Rogers [9].

(9) $\psi=p^{-1} \Gamma p$ for some recursive permutation $p$.

Proof. We consider only the case where $R=N$. (1) is equivalent to (2) by 4.1, since $\Gamma$ is an indexing and $\varnothing$ completes $\Gamma$.

(2) is equivalent to (3) by the definition of universality.

(3) is equivalent to (4) by the results of Cleave [1].

(3) is equivalent to (5) by the transitivity of reducibility and the universality of $\Gamma$. 
(2) implies (6) by 4.4 part (c).

(6) implies (7) by definition.

(7) implies (2) by 5.1 (with $F=\mathrm{id}_{N}$ ).

(1) is equivalent to (8) by the results of Rogers [9] and the fact that $\Gamma=\lambda\langle i, x\rangle\left[\varphi_{i}(x)\right]$.

(8) is equivalent to (9) by a theorem of M. Blum [10, p. 191].

Corollary 5.3. Let $B$ precomplete $\alpha$ and $S$ be an r.e. set such that $\bar{S}=S$, then $S=N$ or $S=\varnothing$ or $S$ is a creative set.

Proof. Suppose $S \neq N$ and $S \neq \varnothing$. Let $F$ be that function which assigns the value 1 to every object in $\alpha[S]$. Then, by 5.1 and 5.2, $F \alpha$ is a creative function, i.e., $S$ is a creative set.

\section{Characterizations.}

THEOREM 6.1. $e$ completes $\alpha$ iff $\alpha$ is a homomorphic image of $\varphi^{*}$ with $e$ as the induced image of $\varnothing$.

Proof. If such a homomorphism exists, then $e$ completes $\alpha$, by 4.4 part (d), since $\varnothing$ completes $\varphi^{*}$.

Suppose that $e$ completes $\alpha$. Then $\alpha \cong E_{\alpha}^{e}$ by 3.2. But $\mathrm{id}_{N}: \Gamma^{*} \rightarrow E_{\alpha}^{e}$ with $e$ as the induced image of $\varnothing$ by 4.6. Hence there is a homomorphism from $\Gamma^{*}$ to $\alpha$ whose induced function assigns the value $e$ to $\varnothing$. Thus we need only show that $\Gamma^{*}$ is a homomorphic image of $\varphi^{*} ; \varnothing$ will be the induced image of $\varnothing$, since, by Corollary 2.2 part (c), only $\varnothing$ completes $\Gamma^{*}$. Let $\psi$ denote $\lambda x\left[\varphi_{x}(1)\right]$. Then $\mathrm{id}_{N}: \varphi^{*} \rightarrow \psi^{*}$, since $\psi^{*}(x)=\psi^{*}(y)$ whenever $\varphi_{x}^{*}=\varphi_{y}^{*}$. Thus, by 4.4 part (d), $\varnothing$ completes $\psi^{*}$, so by $5.2, \psi^{*} \cong \Gamma^{*}$. Thus $\Gamma^{*}$ is a homomorphic image of $\varphi^{*}$.

THeOREM 6.2 (MAL'CEV). A class $\mathscr{S}$ of subsets of $N$ has an $R$-completed r.e. enumeration iff it has an r.e. enumeration and $R$ is a least element of $\mathscr{S}$ with respect to inclusion (i.e., $R \in \mathscr{S}$ and $R=\bigcap \mathscr{S}$ ).

Proof. Suppose $\alpha$ is an $R$-completed r.e. enumeration of $\mathscr{S}$. Let $n \in R$ and let $S=\{x \mid n \in \alpha(x)\}$. Clearly $S$ is r.e. and $S=\bar{S}$. But $\alpha^{-1}[\{R\}] \subset S$. Hence $S=\bar{S}=N$, by 2.2(b), since $\alpha^{-1}[\{R\}]$ precompletes $\alpha$. So for every $x, n \in \alpha(x)$. Thus for every $x$, $R \subset \alpha(x)$ (i.e., $R$ is a least element in $\mathscr{S}$ ).

Suppose, conversely, that $\alpha$ is an r.e. enumeration of $\mathscr{S}$ and $R$ is least in $\mathscr{S}$; in such a case $R$ is clearly r.e. Let $S=\{\langle x, y\rangle \mid$ either $\Gamma(x)$ is defined and $y \in \alpha \Gamma(x)$ or $y \in R$. Clearly $S$ is r.e. If $\Gamma(x)$ is defined, then $S_{x}=R \cup \alpha \Gamma(x)=\alpha \Gamma(x)=E_{\alpha}^{R}(x)$. If $\Gamma(x)$ is not defined $S_{x}=R=E_{\alpha}^{R}(x)$. Thus $E_{a}^{R}$ is the row sequence of $S$. Hence $E_{\alpha}^{R}$ is r.e. But $E_{\alpha}^{R}$ is obviously an enumeration of $\mathscr{S}$, and $R$ completes $E_{\alpha}^{R}$ by 4.6.

In Lachlan [3] universal sequences (respectively, retracts of $W^{*}$ ) are called indexings (respectively, standard enumerations). A class $\mathscr{S}$ is said to be indexable (respectively, standard) iff it admits an indexing (respectively, standard sequence) as an enumeration. Moreover, $\mathscr{S}$ is said to be uniquely indexable iff it indexable and its indexings constitute an isomorphism class. 
From Lemma 4.1 we see that if $\mathscr{S}$ has a precompleted indexing, then it is uniquely indexable. Thus, in view of Theorem 6.2 , a class $\mathscr{S}$ having a least element under inclusion is uniquely indexable iff it is indexable.

From Lachlan [3, Theorem 1.5] we see that there are classes which are indexable but not uniquely indexable; thus by 4.1 there are indexings which are not precompleted.

From [3, Theorem 1.6] we see that there are completed r.e. sequences which are not indexings. For example, $\{\varnothing,\{0\},\{0,1\},\{0,1,2\}, \ldots\}$ has a least element, namely $\varnothing$, and clearly admits an r.e. enumeration, thus by 6.2 it admits a $\varnothing$ completed r.e. enumeration; but it is not indexable (by [3, Theorem 1.6]) since it is not closed under unions of monotonic r.e. sequences.

From Lemmas 4.2 and 4.3 we see that $\mathscr{S}$ is standard iff it has a precompleted uniformly $W^{*}$-universal enumeration. An elementary argument shows that $\alpha$ is uniformly $W^{*}$-universal if and only if it is a uniform indexing in the sense that there exists a recursive function $f$ such that $\varphi_{f(x)}:\left(W_{x}\right)^{*} \leqq \alpha$ whenever $\operatorname{Rng}\left(W_{x}\right)^{*}$ $\subset \operatorname{Rng} \alpha$. Thus by Theorem 6.2 an r.e. sequence whose range contains a least element is standard if and only if it is a uniform indexing. The question of the existence of a standard class having no least element was posed in [2] and answered affirmatively in [3]. This automatically provided an affirmative answer to the question of the existence of a precompleted sequence which is not completed; for any standard enumeration is precompleted since it is a retract of $W$, but in order to be completed its image must have a least element.

The following theorem characterizes the precompleted sequences and provides a simpler example of a standard enumeration of a class having no least element.

THEOREM 6.3. Let $\approx$ denote the smallest equivalence relation on $N$ that contains the graph of $\Gamma$, and let $\Delta$ denote the corresponding sequence of equivalence classes. Then $\Delta$ is a standard enumeration, and for any $B$ and $\alpha, B$ precompletes $\alpha$ if and only if there is a homomorphism $h: \Delta \rightarrow \alpha$ such that $h[Q] \subset B$.

Proof. Suppose that $B$ precompletes $\alpha$. Then by Lemma 3.2 there is a recursive permutation $p$ which is an $\alpha / B$-extension of $\Gamma$. But $\Gamma p^{-1}$ is universal. So by Proposition 5.2 part (9), there is a recursive permutation $h$ such that $\Gamma p^{-1}=h \Gamma h^{-1}$ (i.e. $\left.\Gamma p^{-1} h=h \Gamma\right)$; thus when $\Gamma(x)$ is defined $\alpha h \Gamma(x)=\alpha \Gamma p^{-1} h(x)=\alpha p p^{-1} h(x)=\alpha h(x)$. Thus the equivalence relation induced on $N$ by $\alpha h$ contains the graph of $\Gamma$; hence it contains $\approx$. So $\alpha h(x)=\alpha h(y)$ whenever $\Delta(x)=\Delta(y)$; hence $\mathrm{id}_{N}: \Delta \rightarrow \alpha h$; hence $h: \Delta \rightarrow \alpha$. Furthermore, if $x \in h[Q]$, then $h \Gamma h^{-1}(x)$ is undefined and hence $\Gamma p^{-1}(x)$ is undefined (i.e. $x \in p[Q]$ ). Thus $h[Q]$ is a subset of $p[Q]$ which, in turn, is a subset of $B$.

To show, conversely, that if such a permutation $h$ exists then $B$ precompletes $\alpha$, it suffices (by Lemma 4.4 part (d)) to show that $Q$ precompletes $\Delta$. But this is obviously true, since $\mathrm{id}_{N}$ is a $\Delta / Q$-extension of $\Gamma$. 
$\Delta$ is r.e., hence reducible to $W^{*}$, since $\Delta$ is the row sequence of $\{\langle x, y\rangle \mid x \approx y\}$ which is equal to $\left\{\langle x, y\rangle \mid\right.$ there exists a finite sequence $z_{1}, \ldots, z_{n}$ such that $x=z_{1}$, $y=z_{n}$, and for all $i \in\{1, \ldots, n-1\}, z_{i}=z_{i+1}$ or $z_{i}=\Gamma\left(z_{i+1}\right)$ or $\left.\Gamma\left(z_{i}\right)=z_{i+1}\right\}$.

Now let $\psi$ be a selector for $\left\{\langle i, x\rangle \mid x \in W_{i}\right\}$. Then, when $W_{i} \neq \varnothing, \psi(i)$ is defined and $\psi(i) \in W_{i}$. But if $W_{i}$ is a $\approx$-equivalence class (i.e. $W_{i} \in \operatorname{Rng} \Delta$ ), then $W_{i} \neq \varnothing$, hence $\psi(i) \in W_{i}$, so $\Delta(\psi(i))=W_{i}$. Thus $\psi$ is a subretraction of $W^{*}$ to $\Delta$. But $\Delta$ is precompleted; hence it must be a retract of $W^{*}$.

\section{REFERENCES}

1. J. P. Cleave, Creative functions, Z. Math. Logik Grundlagen Math. 7 (1961), 205-212. MR 25 \#3829.

2. A. H. Lachlan, Standard classes of recursively enumerable sets, Z. Math. Logik Grundlagen Math. 10 (1964), 23-42. MR 28 \#4994.

3. - On the indexing of classes of recursively enumerable sets, J. Symbolic Logic 31 (1966), 10-22. MR 34 \#2451.

4. A. I. Mal'cev, Totally enumerated sets, Algebra i Logika Sem. 2 (1963), 4-29. (Russian) MR 34 \#1179.

5. - On the theory of computable families of objects, Algebra i Logika Sem. 3 (1964), 5-31. (Russian) MR 34 \#48.

6. J. Myhill, Creative sets, Z. Math. Logik Grundlagen Math. 1 (1955), 97-108. MR 17, 118.

7. W. E. Ritter, Notation systems and an effective fixed point property, Proc. Amer. Math. Soc. 17 (1966), 390-395. MR 34 \#1181.

8. H. Rogers, Jr., Gödel numberings of partial recursive functions, J. Symbolic Logic 23 (1958), 331-341. MR 21 \#2585.

9. - On universal functions, Proc. Amer. Math. Soc. 16 (1965), 39-44. MR 30 \#1932.

10. - Theory of recursive functions and effective computability, McGraw-Hill, New York, 1967. MR 37 \#61.

11. R. M. Smullyan, Theory of formal systems, Ann. Math. Studies, no. 47, Princeton Univ. Press, Princeton, N. J., 1961. MR 22 \#12042.

Department of Mathematics, University of California, Riverside, California 92502 